\title{
El empoderamiento como estrategia gerencial en las organizaciones educativas
}

\author{
Empowerment as a management strategy in educational organizations
}

\begin{tabular}{|c|c|c|c|}
\hline$a \rightarrow$ & $\begin{array}{l}\text { Josefina Lucia Peñuela Navarro } \\
\text { anofesoj54@hotmail.com } \\
\text { Código ORCID: 0000-0002-6777-8496 } \\
\text { Universidad del Zulia, Venezuela }\end{array}$ & $a \rightarrow$ & $\begin{array}{l}\text { Maigualida Bejas } \\
\text { maigualidabejas@yahoo.com } \\
\text { Código ORCID: 0000-0003-3030-9298 } \\
\text { Universidad del Zulia, Venezuela }\end{array}$ \\
\hline$\square \rightarrow$ & $\begin{array}{l}\text { Mairene Argelia Veja Monzant } \\
\text { vejamairene@gmail.com } \\
\text { Código ORCID: 0000-0003-1859-1948 } \\
\text { Universidad del Zulia, Venezuela }\end{array}$ & & \\
\hline
\end{tabular}

Artículo recibido en febrero 2019 / Arbitrado en marzo 2019 / Publicado en mayo 2019

\section{Resumen}

El objetivo general de esta investigación fue analizar el empoderamiento como estrategia gerencial en los docentes de las organizaciones educativas del Municipio de Sabanalarga. Se enmarcó como descriptiva con un diseño de campo, transeccional y no experimental. La población estuvo constituida por ochenta y ocho unidades informantes, que fueron escogidos de la siguiente forma: cinco directivos y ochenta y tres docentes de las instituciones educativas San Pedro Claver y Fernando Hoyos Ripoll, ambas ubicadas en Sabanalarga Atlántico (Colombia). Para la recolección de la información se utilizó la técnica de la observación mediante encuesta, se elaboró un instrumento tipo cuestionario. En el análisis de los datos se empleó la estadística descriptiva. Se concluyó que casi nunca el empoderamiento está representado por factores y aspectos del empoderamiento, al igual que las estrategias gerenciales casi nunca están representadas por las acciones estratégicas y las estrategias participativas.

\section{Abstract}

The general objective of this research was to analyze empowerment as a management strategy in teachers of educational organizations in the Municipality of Sabanalarga. It is framed as descriptive, with a field design, transectional and non-experimental. The population consisted of eightyeight informant units, which were chosen as follows: five managers and eighty-three teachers from the San Pedro Claver and Fernando Hoyos Ripoll educational institutions, both located in Sabanalarga Atlántico (Colombia). For the collection of the information, the observation technique was used through a survey, a questionnaire-type instrument was developed. For the analysis of the data, descriptive statistics were used. It was concluded that empowerment is almost never represented by factors and aspects of empowerment, just as management strategies are almost never represented by strategic actions and participatory strategies.
Palabras clave:

Empoderamiento; estrategia gerencial; organizaciones educativas
Keywords:

Empowerment; management strategy; educational organizations 


\section{INTRODUCCIÓN}

Las últimas tendencias de la gerencia moderna a nivel mundial han hecho que las organizaciones se encuentren explorando diferentes opciones para optimizar la gestión de los directores que las conducen. Esto se extiende a las instituciones educativas, las cuales vienen accediendo a la inserción del conglomerado que las integra. También se suma la comunidad de los alrededores con la intención de fortalecer las actividades. Por ello cada director necesita tener un perfil que le permita llevar a cabo acciones de gestión para direccionar a su talento humano, donde se tome en consideración los aspectos principales del capital humano y las competencias individuales de los mismos.

En efecto, internacionalmente esta forma de gestión ha generado grandes transformaciones en las organizaciones educativas, donde la figura del director ha dejado de ser el conductor de las actividades, encargado de trabajar con la transmisión de información y tramitación de documentos a un actor accesible, comunicador, generador de empatía y honestidad, cuyo perfil se encuentra dirigido a desarrollar habilidades, destrezas y valores, conducentes a facilitar las acciones en su gestión, enmarcadas en la efectividad. Sin embargo, aparentemente estos desafíos no se están asumiendo a cabalidad.

Dentro de esta concepción, el gerente es el único con poder transformador de los sistemas dentro de las organizaciones educativas, pues en la sociedad del siglo XXI, quienes gestan el cambio así como el éxito en las organizaciones son los líderes poseedores de la habilidad para comprender aspectos como la incertidumbre y la complejidad; además, deben tener capacidad de generar nuevas ideas para responder con asertividad a las situaciones que se planteen.

De esa forma, haciendo una revisión del contexto en análisis, se tiene que Colombia no es ajena a las exigencias del sector educativo, donde actualmente se considera a las personas el activo más apreciado que tienen las organizaciones, por lo que deben asumir el desafío de desarrollar políticas y estrategias que aumente la motivación de los docentes, optimizando el clima laboral en las mismas. Siendo el conocimiento y la creatividad de parte de los docentes fundamentales para alcanzar las metas de las organizaciones, que deben ir alineados con los objetivos personales de cada uno de los miembros de la comunidad educativa.

Por consiguiente, el gerente educativo recurre a acciones estratégicas para comprometer al personal de la institución en la calidad de la enseñanza, conformando equipos de trabajo dispuestos a contribuir con la innovación, el cambio, la calidad y el incremento de la productividad educativa a fin de reducir las debilidades en las pautas del comportamiento laboral, puesto que las organizaciones escolares actualmente están orientadas hacia el conocimiento colectivo para cuestionar los procesos que se están llevando a cabo en las mismas, todo ello porque estas instituciones deben enfocarse hacia el rendimiento para responder a las demandas actuales de la sociedad.

A pesar de ello, en América Latina y en Colombia pareciera que las estrategias gerenciales necesitan un nuevo enfoque para alcanzar los propósitos de la institución escolar, por tanto es preciso la participación del director desde un punto de vista estratégico a fin de que su Plan Decenal (2018) proponga dentro de su objetivo:

Actualizar y direccionar los programas formativos hacia las necesidades y demandas del sistema productivo nacional, con el fin garantizar la formación técnica, profesional y ocupacional del trabajo, fomentando en cada esfuerzo los valores y el sentido crítico en la organización para el trabajo liberador. (p. 17) 
De allí su deber de orientarse a la productividad, mantener el estímulo al compromiso en el logro de objetivos, desarrollar en los docentes su sentido de responsabilidad y gestionar el desarrollo del talento humano con el propósito de que en las instituciones educativas se logre la innovación, el cambio, la calidad y la productividad para satisfacer las necesidades de los procesos educativos, atendiendo de igual forma las necesidades de formación y capacitación requeridas.

Sin embargo, en las instituciones de educación colombianas, específicamente la I.E Fernando Hoyos Ripoll y San Pedro Claver, posiblemente se presentan síntomas relacionados a la toma decisiones y su centralización, puesto que no consideran la opinión de los docentes a la hora de hacer los proyectos, poseen bajos niveles de cualificación del personal que integra la organización educativa, la misión y visión se hace a puerta cerrada con un pequeño grupo, y las informaciones se manejan para un grupo minoritario. Igualmente sucede con la falta de cambios innovadores en las funciones de los docentes y en la inexistencia de un supervisor como facilitador, el cual establezca los límites de los equipos de trabajo para que a su vez los forme con técnicas y habilidades que más adelante contribuirán a la solución de problemas.

Es por esas razones que se identificaron algunas causas originarias de los síntomas descritos anteriormente, las cuales son: a) Trabajo repetitivo y sin importancia; b) confusión entre los docentes; c) falta de confianza; d) falta de contribución en las decisiones que se toman en la institución; e) no conocen los docentes si su desempeño es óptimo o no; f) no conocen con claridad los aspectos estructurales - organizacionales de la institución; g) tienen poco tiempo para resolver los problemas que se puedan presentar dentro de la institución; h) no se les da crédito a los docentes por sus ideas o esfuerzos realizados; e i) no se evidencia desarrollo de competencias del hacer en algunos miembros de la organización.

Por tanto, dichas organizaciones del sector educativo develan la necesidad de implementar acciones para ser competitivas y poder mantenerse ante las exigencias de la sociedad. En tal sentido, el propósito del empoderamiento como estrategia gerencial consiste en implementar y motivar al personal hacia el logro de las metas, alcanzando una alta fortaleza en la información de los servicios educativos que se ofrecen, dando como resultado seguridad en el cargo, amplio entrenamiento, sistema de recompensas, información compartida, así como el fenómeno de la equidad.

En tal sentido, el objetivo central de investigación fue analizar el empoderamiento como estrategia gerencial en los docentes de las organizaciones educativas del Municipio de Sabanalarga. Los objetivos específicos fueron: Identificar los factores presentes del empoderamiento en los docentes de las organizaciones educativas del Municipio de Sabanalarga; determinar los aspectos a empoderar en las organizaciones educativas del Municipio de Sabanalarga; y, por último, describir las acciones estratégicas gerenciales del directivo en las organizaciones educativas del Municipio de Sabanalarga.

\section{MÉTODO}

El diseño de investigación se consideró como un procedimiento a seguir por el investigador en atención al problema o hecho a investigar. En el presente estudio se utilizó un diseño no experimental, transaccional o transversal y de campo.

Se estimó también como transeccional, transversal sobre la base de las ideas expuestas, el diseño de estas investigaciones busca describir y medir la variable en estudio, empoderamiento como estrategia gerencial en las organizaciones educativas, 
en un tiempo único. En esta perspectiva, la investigación planteada se considera de campo, por cuanto la información será tomada específicamente en las organizaciones educativas colombianas, con miras a analizar el empoderamiento como estrategia gerencial en dichas instituciones.

El muestreo se basó en una selección, cuyos elementos son escogidos por la decisión personal del investigador, por situaciones de conveniencia y facilidad de acceso, y porque allí se evidenció la problemática. Así, en el presente caso, la población de esta investigación estuvo conformada por ochenta y ocho unidades informantes, que fueron escogidos de la siguiente forma: cinco directivos y ochenta y tres docentes de las instituciones educativas Fernando Hoyos Ripoll y San Pedro Claver, ambas ubicadas en Sabanalarga Atlántico (Colombia), tal como se muestra en el siguiente Tabla 1.

Tabla 1. Distribución de la población

\begin{tabular}{lccc}
\hline Organizaciones Educativas & $\mathbf{N}^{\circ}$ Directivos & $\mathbf{N}^{\circ}$ Docentes & Total \\
\hline Fernando Hoyos Ripoll & 3 & 44 & 47 \\
San Pedro Claver & 2 & 39 & 41 \\
Total & 5 & 83 & 88 \\
\hline
\end{tabular}

Fuente: Nómina de los planteles (2019).

La técnica e instrumento de recolección de datos fue el cuestionario. Para efectos de recopilar información acerca del empoderamiento como estrategia gerencial en las organizaciones educativas, se diseñó un cuestionario de cuarenta y dos ítems versionado, tanto para los directivos como para el personal docente. Dicho instrumento se construyó con preguntas cerradas, escala de Likert, con cinco opciones de respuesta: Siempre, Casi Siempre, Algunas veces, Casi Nunca y Nunca. Ver Tabla 2:

Tabla 2. Ponderación de las respuestas

\begin{tabular}{cc}
\hline Ponderación & Alternativas de Respuestas \\
\hline 5 & Siempre \\
4 & Casi Siempre \\
3 & Algunas veces \\
2 & Casi Nunca \\
1 & Nunca \\
\hline
\end{tabular}

De igual forma, se aplicó una prueba piloto, la cual se le administró a un mismo número de población similar a la seleccionada, pero en otra organización educativa, para determinar la confiabilidad del instrumento, para demostrar una mayor veracidad con respecto al tipo de instrumento a aplicar y el resultado que este arrojó.

Dado que el instrumento estuvo compuesto por el escalamiento de tipo
Likert, el método para calcular su confiabilidad fue la prueba piloto, que consiste en aplicar el instrumento a una población distinta a la estudiada, pero con características que le asemejan. Del mismo modo, la ecuación que se administró fue el coeficiente Alfa de Cronbach, la cual es la más precisa para estos casos y se muestra a continuación 
Tabla 3. Escala para la valoración del coeficiente de confiabilidad

\begin{tabular}{ll}
\hline Rangos & Magnitud \\
\hline o,81 a 1,00 & Muy alta \\
0,61 a 0,80 & Alta \\
0,41 a 0,60 & Moderada \\
0,21 a 0,40 & Baja \\
0,01 a 0,20 & Muy baja \\
\hline
\end{tabular}

Fuente: Ruiz (2002) y Peñuela, Bejas y Vejas (2019).

Una vez aplicada la prueba piloto, se procedió a tabular los datos y aplicar la fórmula expuesta anteriormente, para lo cual se obtuvo el coeficiente de confiabilidad $r_{\text {tt }}$ $=0,976$ cuya interpretación es de magnitud muy alta, estableciéndose así, que el instrumento diseñado para la presente investigación fue altamente confiable, de acuerdo al baremo establecido.

\section{RESULTADOS}

El análisis de los resultados se presenta en tablas que resumen el comportamiento de los indicadores en relación a las preguntas y respuestas establecidas, la cual se confrontan con las bases teóricas. La Tabla 4 muestra los resultados que dieron respuesta al primer objetivo específico, el cual buscó identificar los factores presentes del empoderamiento en las organizaciones educativas San Pedro Claver y Fernando Hoyos Ripoll del Municipio de Sabanalarga. La Variable: Empoderamiento como Estrategia Gerencia.

Tabla 4. Dimensión: Factores de Empoderamiento

\begin{tabular}{lcccccccccccccccccccccc}
\hline \multicolumn{1}{c}{ Alternativas } & \multicolumn{1}{c}{ S } & \multicolumn{1}{c}{ Indicador } & Dir. & $\%$ & Doc. & $\%$ & Dir. & $\%$ & Doc. & $\%$ & Dir. & $\%$ & Doc. & $\%$ & Dir. & $\%$ & Doc. & $\%$ & Dir. & $\%$ & Doc. & $\%$ \\
\hline Falta de confianza & 3 & 0,0 & 3 & 35,28 & 2 & 0,00 & 8 & 25,73 & 0 & 0.00 & 21 & 20,45 & 0 & 60,00 & 30 & 18,54 & 0 & 40,00 & 21 & 0,00 \\
Empatía & 2 & 40,00 & 2 & 2,41 & 3 & 60,00 & 7 & 8,43 & 0 & 0,00 & 25 & 30,12 & 0 & 0,00 & 39 & 46,99 & 0 & 0,00 & 10 & 12,05 \\
Toma de decisiones & 3 & 60,00 & 4 & 4,82 & 1 & 20,00 & 11 & 13,25 & 1 & 20,00 & 27 & 32,53 & 0 & 0,00 & 35 & 42,17 & 0 & 0,00 & 6 & 7,23 \\
Capacitación & 3 & 60,00 & 3 & 3,61 & 2 & 40,00 & 10 & 12,05 & 0 & 0,00 & 24 & 28,92 & 0,00 & 33 & 39,76 & 0 & 0,00 & 13 & 15,66 \\
\hline \multicolumn{1}{c}{$\%$ de Grupo } & $\mathbf{4 0 , 0 0}$ & $\mathbf{1 1 , 5 3}$ & $\mathbf{3 0 , 0 0}$ & $\mathbf{1 4 , 8 7}$ & $\mathbf{5 , 0 0}$ & $\mathbf{2 8 , 0 0}$ & $\mathbf{1 5 , 0 0}$ & $\mathbf{3 6 , 8 6}$ & & $\mathbf{1 0 , 0 0}$ & $\mathbf{8 , 7 3}$ \\
\hline
\end{tabular}

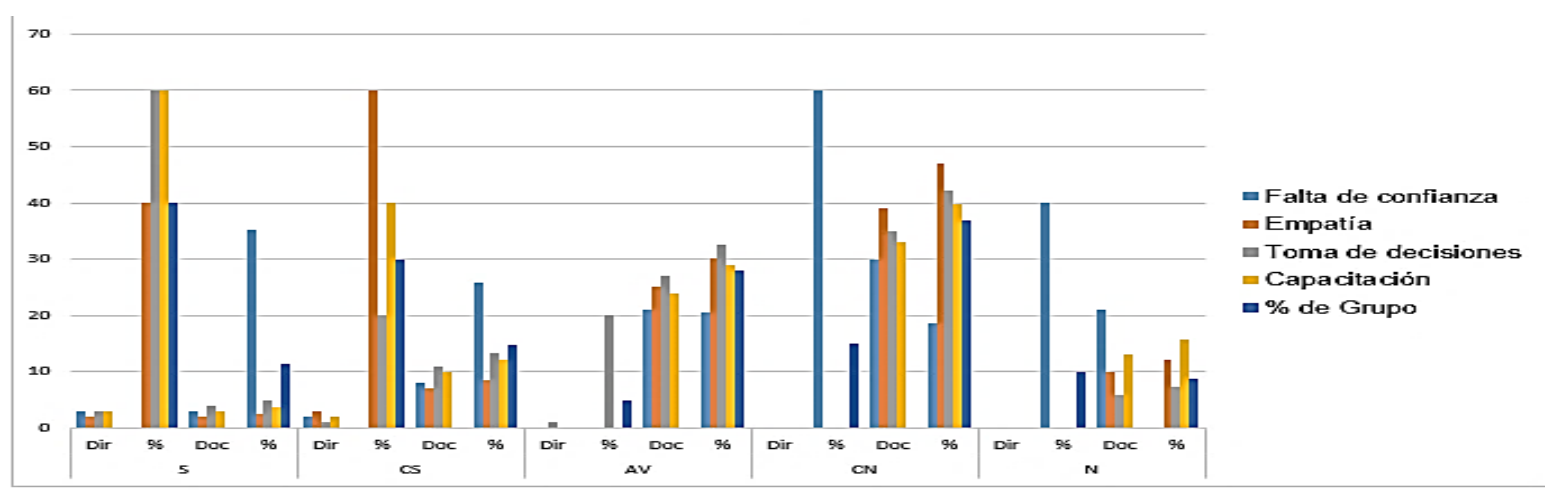

Gráfico 1. Factores de empoderamiento 
Iniciando con el primer indicador, falta de confianza, los directivos encuestados opinaron en un $60,00 \%$, que Casi nunca existe falta de confianza como factor de empoderamiento; Nunca 40,00\%; Siempre, Casi siempre y Algunas veces o,oo\%. Los docentes al respecto expresaron en un $35,28 \%$, que Siempre se da la falta de confianza; Casi siempre 25,73\%; Algunas veces 20,45\%; Casi nunca $18,54 \%$ y Nunca o,oo\%.

Los docentes a su vez opinaron que los directivos siempre dejan de fortalecer las relaciones de amistad entre sus compañeros de trabajo, dejando de incentivarlos a dar lo mejor de sí y, de esta manera, poder delegar responsabilidades en el personal a su cargo.

Para el indicador empatía, los directivos respondieron en un $60,00 \%$, que Siempre se da la empatía; Casi siempre 40,00\%, Algunas veces, Casi nunca y Nunca o,oo\%. Los docentes al respecto expresaron en un 46,99\% que Casi nunca existe empatía; Algunas veces 30,12\%; Nunca 12,05\%; Siempre $2,42 \%$ y Casi siempre $8,43 \%$ Los docentes en el mismo sentido expresaron que los directivos casi nunca perciben las necesidades del personal, al igual que los sentimientos no expresados verbalmente comprendiendo las perspectivas de los demás.

Cabe destacar que dicha percepción se contradice con lo expuesto por Albrecht (2006), quien señala que la empatía es una connotación habitual de simpatizar o identificarse con otras personas. Considerándose así a la empatía como un estado de conexión y sentimientos compartidos que se experimentan con otra persona que origina una base para la interacción positiva y la cooperación.

Abordando el indicador toma de decisiones, los directivos infirieron en un $60,00 \%$ que Casi siempre toman decisiones; Siempre y Algunas veces 20,00\%; Casi nunca y nunca $0,00 \%$. Los docentes al respecto opinaron en un $42,17 \%$ que Casi nunca los directivos toman decisiones en la institución; Algunas veces 32,53\%; Casi siempre 13,25\%; Siempre $4,82 \%$ y Nunca $7,23 \%$. Los docentes, en este sentido, infirieron que los directivos Casi nunca alcanzan sus acuerdos de manera mutua entre todos los miembros del equipo de trabajo, omitiendo la discusión de los puntos de vista hasta que todos estén de acuerdo dejando así de tomar en cuenta la opinión de todos para la toma de decisiones requeridas por la institución. Según Faintein (2008) describe que la toma de decisiones refiere al desarrollo de los equipos, vinculado a la toma de decisión, este aspecto, consiste en alcanzar un acuerdo mutuo entre todos los miembros del equipo, al momento de participar en la toma de decisiones, deben discutir los puntos de vista hasta que todos estén de acuerdo.

Finalmente para el indicador capacitación, los directivos expresaron en un $60,00 \%$ que Siempre existe capacitación, Casi siempre 40,00\%, Algunas veces, Casi nunca y Nunca 0,oo\%. Los docentes al respecto expresaron en un $39,76 \%$ que los directivos Casi nunca fomentan la capacitación, Algunas veces $28,92 \%$, Nunca $15,66 \%$, Casi siempre $12,05 \%$ y Siempre 3,61\%.

Los docentes enunciaron que los directivos Casi nunca desarrollan acciones encaminadas a mejorar las habilidades del personal, sin propiciar actividades que perfeccionen al personal en su puesto de trabajo y dejando de incentivar al personal a satisfacer la necesidad de la institución educativa de nuevos conocimientos.

La Tabla 5 pretendió dar respuesta al segundo objetivo específico, el cual persiguió describir las acciones estratégicas gerenciales del directivo en las organizaciones educativas San Pedro Claver y Fernando Hoyos Ripoll del Municipio de Sabanalarga. 
Tabla 5. Dimensión: Aspectos de empoderamiento

\begin{tabular}{|c|c|c|c|c|c|c|c|c|c|c|c|c|c|c|c|c|c|c|c|c|}
\hline \multirow{2}{*}{$\begin{array}{c}\text { Alternativas } \\
\text { Indicador }\end{array}$} & \multicolumn{4}{|c|}{$\mathrm{S}$} & \multicolumn{4}{|c|}{ CS } & \multicolumn{4}{|c|}{ AV } & \multicolumn{4}{|c|}{$\mathrm{CN}$} & \multicolumn{4}{|c|}{$\mathbf{N}$} \\
\hline & Dir. & $\%$ & Doc. & $\%$ & Dir. & $\%$ & Doc. & $\%$ & Dir. & $\%$ & Doc. & $\%$ & Dir. & $\%$ & Doc. & $\%$ & Dir. & $\%$ & Doc. & $\%$ \\
\hline Creencias & 2 & 40,00 & 3 & 3,61 & 1 & 20,00 & 12 & 14,46 & 2 & 40,00 & 22 & 26,51 & 0 & 0,00 & 30 & 36,14 & 0 & 0,00 & 16 & 19,28 \\
\hline Valores y aptitudes & 3 & 60,00 & 5 & 6,02 & 1 & 20,00 & 10 & 12,05 & 1 & 20,00 & 23 & 27,71 & 0 & 0,00 & 32 & 38,55 & 0 & 0,00 & 13 & 15,66 \\
\hline Conocimiento & 3 & 60,00 & 5 & 6,02 & 2 & 40,00 & 21 & 25,30 & 0 & 0,00 & 21 & 25,30 & 0 & 0,00 & 29 & 34,94 & 0 & 0,00 & 7 & 8,43 \\
\hline$\%$ de Grupo & & 53,33 & & 5,22 & & 26,67 & & 17,27 & & 20,00 & & 26,51 & & 0,00 & & 36,55 & & 0,00 & & 14,46 \\
\hline
\end{tabular}

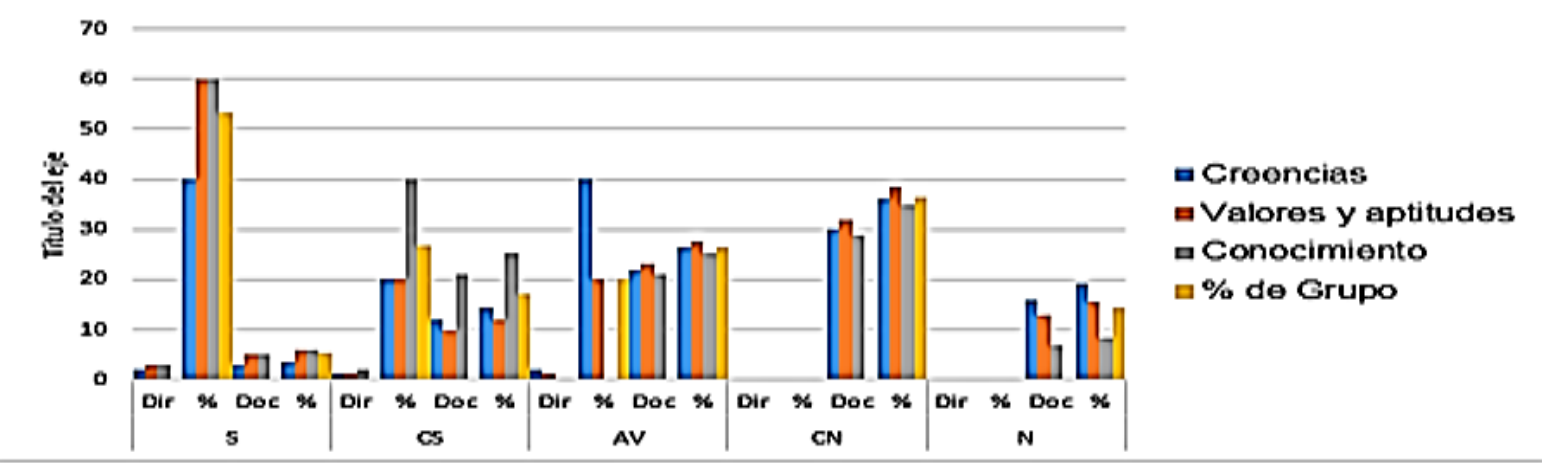

Gráfico 2. Aspectos de empoderamiento

Para el indicador creencias, los directivos expresaron en un 40,00\%, que Siempre y Algunas veces existe la creencia como aspecto de empoderamiento; Casi siempre 20,00\%; Casi nunca y Nunca o,oo\%. Por su parte, los docentes opinaron en un $36,14 \%$ que los directivos Casi nunca expresan creencia; Algunas veces 26,51\%; Nunca 19,28\%; Casi siempre $14,46 \%$ y Siempre o,oo\%. Los docentes a su vez expresaron que los directivos Casi nunca aceptan aquellas ideas integrales como válidas, sin utilizar las creencias de sus subordinados que sirven para expresar la realidad de la institución educativa, lo que dificulta la configuración de los valores de la institución educativa.

La percepción dada por los docentes contradijo lo postulado por Capriotti (2009), quien describe que las creencias son el conjunto de presunciones básicas compartidas por los miembros de la organización sobre los diferentes aspectos y cuestiones de la organización.
Para el indicador valores y aptitudes, los directivos encuestados opinaron en un 6o,00\% que Siempre; Casi siempre y Algunas veces 20,00\%; Casi nunca y Nunca $0,00 \%$. Los docentes al respecto opinaron en un $38,55 \%$ que Casi nunca los directivos expresan valores y aptitudes; Algunas veces 27,71\%; Nunca 15,66\%; Casi siempre 12,05\% y Siempre $6,02 \%$. Según la opinión de los docentes, los directivos Casi nunca manejan una disciplina acorde a las necesidades de la institución educativa, lo que infunde desmotivación para alcanzar sus metas operacionales sintiendo poco compromiso hacia la institución educativa.

Para ello, Capriotti (2009) señala que los valores son el conjunto de principios compartidos por los miembros de la organización en su relación cotidiana dentro de la entidad. Tienen un mayor grado de visibilidad que las creencias, pero no tienen una observación claramente manifiesta. En ese sentido, son importantes porque 
describen lo que es primordial para sus implicados, porque identifican los resultados que la mayoría espera, guían nuestras actuaciones y determinan si nuestras organización tendrá éxito.

Abordando el indicador conocimiento, los directivos encuestados opinaron en un $60,00 \%$ que Siempre demuestran conocimiento; Casi siempre 40,00\%; Algunas veces; Casi nunca y Nunca o,oo\%. Por su parte, los docentes consideraron en un 34,94\% que los directivos Casi nunca demuestran conocimientos; Casi siempre y Algunas veces 25,30\%; Nunca 8,43\% y Siempre $6,02 \%$. Por tal motivo, los docentes infirieron que los directivos casi nunca se esfuerzan en capacitarse para el mejor desempeño de sus funciones, sin generar concursos para mejorar su conocimiento ni realizar actividades de capacitación continua para el desarrollo profesional.

Estas opiniones se alejan de lo expuesto por Chiavenato (2010), quien señala que las personas poseen conocimiento y habilidades con los cuales pueden contribuir a que las organizaciones alcance sus objetivos. Hace énfasis en que existen estrategias aplicadas por las empresas, dirigidas a suministrar conocimiento y adiestramiento al personal y obtener un buen desempeño al momento de delegar autoridad y estos tomen decisiones acertadas, apoyen a sus compañeros y contribuyan para el logro de las metas organizacionales.

\section{La variable: Estrategia gerencial}

En relación a la Tabla 6, la cual pretendió dar respuesta al tercer objetivo específico, que buscó describir las acciones estratégicas gerenciales del directivo en las organizaciones educativas San Pedro Claver y Fernando Hoyos Ripoll del Municipio de Sabanalarga.

Tabla 6. Dimensión: Acciones estratégicas de empoderamiento por indicador

\begin{tabular}{|c|c|c|c|c|c|c|c|c|c|c|c|c|c|c|c|c|c|c|c|c|}
\hline \multirow{2}{*}{$\begin{array}{c}\text { Alternativas } \\
\text { Indicador }\end{array}$} & \multicolumn{4}{|c|}{ S } & \multicolumn{4}{|c|}{ CS } & \multicolumn{4}{|c|}{ AV } & \multicolumn{4}{|c|}{$\mathrm{CN}$} & \multicolumn{4}{|c|}{$\mathbf{N}$} \\
\hline & Dir. & $\%$ & Doc. & $\%$ & Dir. & $\%$ & Doc. & $\%$ & Dir. & $\%$ & Doc. & $\%$ & Dir. & $\%$ & Doc. & $\%$ & Dir. & $\%$ & Doc. & $\%$ \\
\hline Innovación & 3 & 60,00 & 2 & 2,41 & 2 & 40,00 & 7 & 8,43 & 0 & 0,00 & 18 & 21,69 & 0 & 0,00 & 43 & 51,81 & 0 & 0,00 & 13 & 15,66 \\
\hline Cambio & 1 & 20,00 & 2 & 2,41 & 3 & 60,00 & 7 & 8,43 & 1 & 20,00 & 22 & 26,51 & 0 & 0,00 & 36 & 43,37 & 0 & 0,00 & 16 & 19,28 \\
\hline Calidad & 1 & 20,00 & 3 & 3,61 & 3 & 60,00 & 10 & 12,05 & 1 & 20,00 & 24 & 28,92 & 0 & 0,00 & 40 & 48,19 & 0 & 0,00 & 6 & 7,23 \\
\hline Productividad & 3 & 60,00 & 6 & 7,23 & 2 & 40,00 & 16 & 19,28 & 0 & 0,00 & 22 & 26,51 & 0 & 0,00 & 28 & 33,73 & 0 & 0,00 & 11 & 13,25 \\
\hline$\%$ de Gru & & 40,00 & & 3,92 & & 50,00 & & 12,05 & & 10,00 & & 25,90 & & 0,00 & & 44,28 & & 0,00 & & 13,86 \\
\hline
\end{tabular}

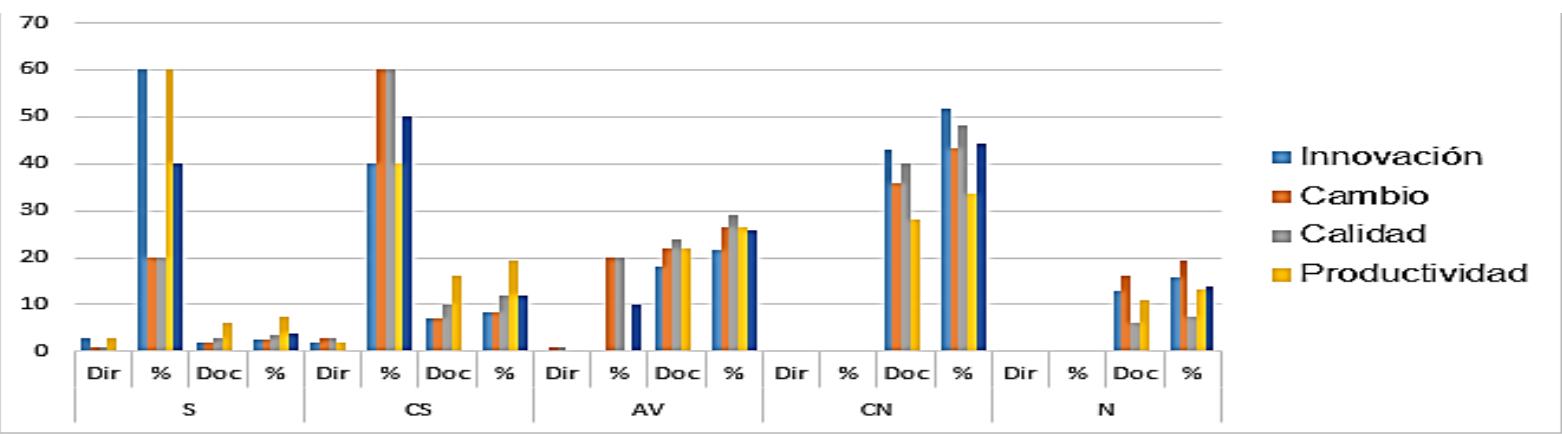

Gráfico 3. Acciones estratégicas de empoderamiento 
Dando inicio a la tercera dimensión, la cual comienza con el indicador innovación, los directivos opinaron en un $60,00 \%$, que Siempre existe innovación; Casi siempre 40,00\%; Algunas veces, Casi nunca y Nunca o,oo\%. Los docentes al respecto opinaron en un $51,81 \%$ que Casi nunca los directivos apoyan la innovación; Algunas veces 21,69\%, Nunca 15,66\%, Casi siempre $8,43 \%$ y Siempre 2,41\%.

Para los docentes, los directivos casi nunca generan en la institución nuevas políticas para que se preocupen por innovar las formas de enseñanza, sin promover el aprendizaje de las nuevas tecnologías de información y comunicación como estrategias de enseñanza lo que dificulta la verificación de los logros en los cambios de las estrategias de enseñanza.

En relación al segundo indicador, los directivos encuestados expresaron en un 6o,00\% que Casi siempre existe el cambio; Siempre y Algunas veces 20,00\%; Casi nunca y Nunca $0,00 \%$. Los docentes por su parte opinaron en un $43,37 \%$ que los directivos Casi nunca apoyan el cambio, algunas veces $26,51 \%$, Nunca $19,28 \%$, Casi siempre $8,43 \%$ y siempre $2,41 \%$. Los docentes en el mismo sentido expresaron que los directivos Casi nunca presionan para que se acepten los cambios educativos sin percibir que los docentes sienten temor ante dichos cambios, lo que genera desinterés por propiciar condiciones psicológicas adecuadas para que se generen cambios positivos en la institución.

Según Chiavenato "el cambio constituye el tránsito de un estado a otro. Es la transición de una situación a otra distinta. El cambio representa siempre transformación, alteración, modificación, perturbación, interrupción, fractura, ruptura" (2010, p. 9).

En relación al indicador calidad, los directivos opinaron en un $60,00 \%$, que Casi siempre existe calidad, siempre y algunas veces $20,00 \%$, Casi nunca y nunca $0,00 \%$, los docentes a su vez expresaron en un $48,19 \%$, que Casi nunca los directivos fomentan calidad, algunas veces $28,92 \%$, Casi siempre $12,05 \%$, nunca $7,23 \%$ y siempre $3,61 \%$. Los docentes en ese sentido expresaron que los directivos Casi nunca colaboran con las diversas coordinaciones al momento de tomar decisiones que beneficien la calidad de los procesos educativos, sin controlarlos con el fin de que se mantengan bajo estándares e impidiendo el compartir responsabilidad por la calidad de la educación.

Por lo que esto contradice lo expuesto por Polimeni, Fabozzi y Adelberg (2007), quienes expresan que "la gerencia debe colaborar con los diversos departamentos dentro de la organización cuando se evalúan las políticas y en el momento de tomar las decisiones que afecten la calidad" (p.405).

Para el indicador productividad, los directivos expusieron en un $60,00 \%$ que Siempre fomentan la productividad, Casi siempre 40,00\%, Algunas veces, Casi nunca y Nunca $0,00 \%$. Por su parte, los docentes expresaron en un 33,73\%, que los directivos Casi nunca promueven la productividad, Algunas veces $26,51 \%$, Casi siempre $19,28 \%$, Nunca $13,25 \%$ y Siempre $7,23 \%$. En este sentido los docentes infirieron que los directivos Casi nunca observan si a través de su productividad se alcanzan las metas establecidas, por lo que no aplican instrumentos confiables para verificarla y si esta se adapta a las necesidades de la institución, careciendo de planteamientos que se enfoquen en las necesidades de actualizarse como una vía para mejorar dicha productividad.

En la Tabla 7, la cual pretende dar respuesta a la primera variable empoderamiento, como estrategia gerencial en las organizaciones educativas San Pedro Claver y Fernando Hoyos Ripoll del Municipio de Sabanalarga. 
Tabla 7. Variable Empoderamiento

\begin{tabular}{lcccccccc}
\hline \multirow{2}{*}{ Alternativas } & \multicolumn{3}{c}{ Factores de Empoderamiento } & \multicolumn{3}{c}{ Aspectos de Empoderamiento } \\
& Dir. & $\%$ & Doc. & $\%$ & Dir. & $\%$ & Doc. & $\%$ \\
\hline SIEMRPE & 11 & 55,00 & 12 & 3,61 & 8 & 53,33 & 13 & 5,22 \\
CASI SIEMPRE & 8 & 40,00 & 36 & 10,87 & 4 & 26,67 & 43 & 17,27 \\
A VECES & 1 & 5,00 & 97 & 29,22 & 3 & 20,00 & 66 & 26,51 \\
CASI NUNCA & 0 & 0,00 & 137 & 41,27 & 0 & 0,00 & 91 & 36,55 \\
\hline NUNCA & $\mathbf{0}$ & $\mathbf{0 , 0 0}$ & $\mathbf{5 0}$ & $\mathbf{1 5 , 0 6}$ & $\mathbf{0}$ & $\mathbf{0 , 0 0}$ & $\mathbf{3 6}$ & $\mathbf{1 4 , 4 6}$ \\
\hline
\end{tabular}

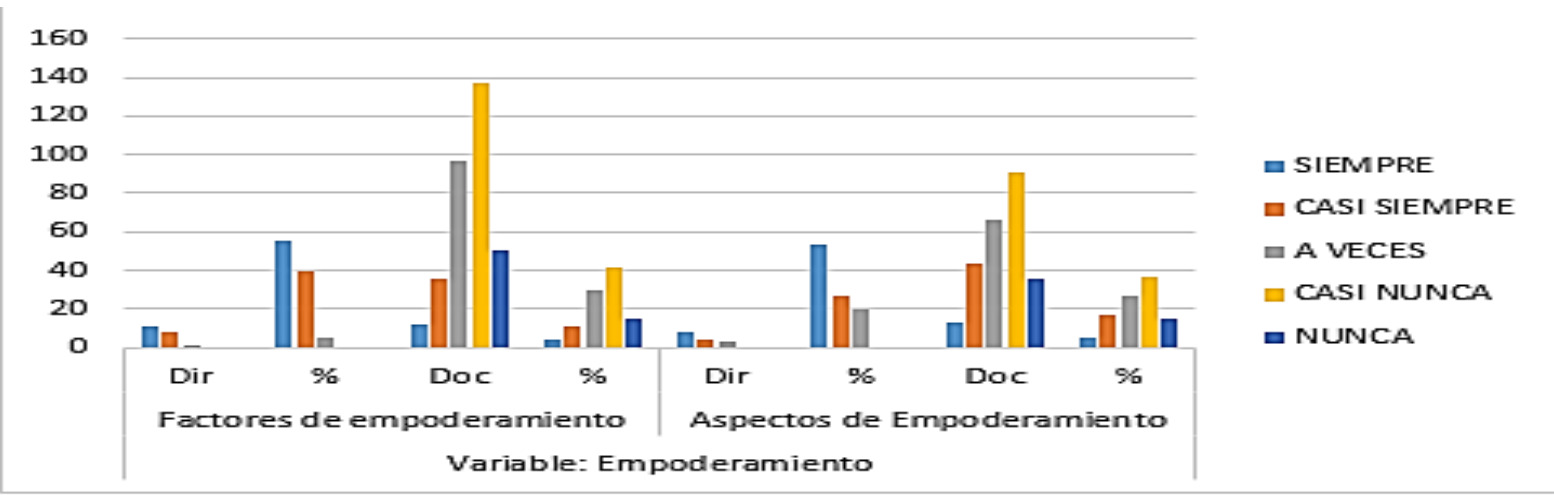

Gráfico 4. Empoderamiento

Para la dimensión factores de empoderamiento los directivos respondieron en un $55,55 \%$ que Siempre están presentes factores como Falta de confianza, Empatía, Toma de decisiones y Capacitación, Casi siempre 40,00\%, Algunas veces 5,00\%, Casi nunca y nunca $0,00 \%$. Los docentes al respecto expresaron en un $41,27 \%$ que los directivos Casi nunca demuestran dichos factores, algunas veces $29,22 \%$, nunca $15,06 \%$, Casi siempre $10,84 \%$ y siempre 3,61\%.Cabe destacar que Newstrom (2011) señala que este modelo engloba nuevas ideas, dejando atrás las teorías ya oxidadas de hacerse rico por medio del trabajo y la motivación, la palabra encaja con los conceptos modernos que dirigen las organizaciones en la actualidad como la mejora continua, la gestión del rendimiento, grupos de trabajos autodirigidos, gestión recompetencias, entre otros; originando una nueva técnica de cohesión laboral, para obtener logros de objetivos comunes.

En relación a la dimensión aspectos de empoderamiento los directivos expresaron en un 53,33\% que siempre toman en cuenta los aspectos como Creencias, Valores y actitudes y Conocimiento, Casi siempre $26,67 \%$, algunas veces $20,00 \%$, Casi nunca y nunca $0,00 \%$. Los docentes a su vez opinaron en un $36,55 \%$ que los directivos Casi nunca demuestran dichos aspectos, algunas veces $26,51 \%$, Casi siempre $17,27 \%$, nunca $14,46 \%$ y siempre 5,22\%.Según según Hellriegel (2009), quien señala que existen evidencias notables que las organizaciones con mayor número de empleados empoderados $\mathrm{o}$ facultados son las que han experimentado mayor crecimiento en el mundo. De allí, que partiendo del concepto de que la dirección es la habilidad para guiar a las personas a un objetivo determinado, el empoderamiento es 
la habilidad del directivo para crear confianza en el trabajador sobre su desempeño.

La segunda variable estrategias gerenciales, para el empoderamiento como estrategia gerencial en las organizaciones educativas San Pedro Claver y Fernando Hoyos Ripoll del Municipio de Sabanalarga, para la dimensión acciones estrategias de empoderamiento, en el primer indicador, los directivos opinaron en un 50,00\% que Casi siempre se cumplen acciones estratégicas como Innovación, Cambio, Calidad y Productividad, siempre 40,00\%, algunas veces $10,00 \%$, Casi nunca y nunca $0,00 \%$. Los docentes a su vez opinaron en un $44,28 \%$ que los directivos Casi nunca ponen en práctica dichas acciones, Algunas veces 29,90\%, Nunca 13,86\%, Casi siempre 12,05\% y Siempre $3,92 \%$. Es importante resaltar que según Abascal (2007) "dichas acciones llamadas estratégicas tienen que ser tan flexibles, que si el entorno en que se están aplicando cambios, dichas acciones también pueden ser cambiadas" (p. 187).

\section{CONCLUSIONES}

En estas líneas finales se expresa que aquellos factores que no están presentes en los directivos como empoderamiento son: Siempre la falta de confianza, seguidos por Casi nunca la empatía, toma de decisiones y capacitación. Los aspectos del empoderamiento en las organizaciones educativas San Pedro Claver y Fernando Hoyos Ripoll del Municipio de Sabanalarga, se basan su desempeño son, Casi nunca las creencias, valores y aptitudes y finalmente el conocimiento.

Seguidamente se afina que para el tercer objetivo específico, el cual buscó describir las acciones estratégicas gerenciales del directivo en las organizaciones educativas San Pedro Claver y Fernando Hoyos Ripoll del Municipio de Sabanalarga, los directivos
Casi nunca ejercen la innovación, ya que no generan nuevas políticas para que se preocupen por innovar las formas de enseñanza, sin promover el aprendizaje de las nuevas tecnologías de información y comunicación como estrategias de enseñanza lo que dificulta la verificación de los logros en los cambios de las estrategias de enseñanza.

Casi nunca el cambio representa una acción estratégica, ya que estos Casi nunca presionan para que se acepten los cambios educativos sin percibir que los docentes sienten temor ante dichos cambios, lo que genera desinterés por propician condiciones psicológicas adecuadas para que se generen cambios positivos en la institución. Igualmente la calidad Casi nunca representa un resultado dado producto de la colaboración grupal en diversas coordinaciones, y al momento de tomar decisiones que beneficien la calidad de los procesos educativos, sin controlarlos con el fin de que se mantengan bajo estándares e impidiendo el compartir responsabilidad por la calidad de la educación.

Para las acciones estratégicas de productividad se resuelve que Casi nunca se alcanzan las metas establecidas, por lo que no aplican instrumentos confiables para verificarla y si esta se adapta a las necesidades de la institución, careciendo de planteamientos que se enfoquen en las necesidades de actualizarse como una vía para mejorar dicha productividad.

Para el cuarto objetivo específico el cual persiguió identificar las estrategias participativas del directivo en las organizaciones educativas San Pedro Claver y Fernando Hoyos Ripoll del Municipio de Sabanalarga, se dedujo que estas estrategias participativas están representadas por Casi nunca el compromiso a responsabilidad y el trabajo en equipo. 
Para el objetivo general el cual enfatizó analizar el empoderamiento como estrategia gerencial en las organizaciones educativas, se concluye que casi nunca el empoderamiento está representado por factores y aspectos del empoderamiento al igual que las estrategias gerenciales casi nunca están representadas por las acciones estratégicas y las estrategias participativas.

En definitiva, se valoró la necesidad de fortalecer las relaciones de amistad entre los compañeros de trabajo, por medio de incentivos, con el fin de dar lo mejor de sí y así poder delegar responsabilidades en el personal su cargo. Crear grupos de diálogo donde se expresen las necesidades del personal, al igual que los sentimientos no expresados verbalmente, para poder comprender las perspectivas de los demás.

Crear mesas de trabajo donde el personal docente establezca acuerdos de manera mutua entre todos los miembros del equipo de trabajo, donde se desarrollen discusiones de los puntos de vista hasta que todos estén de acuerdo. Desarrollar acciones encaminadas a mejorar las habilidades del personal, donde la participación sea un medio para propiciar actividades que perfeccionen al personal en su puesto de trabajo. Resaltar aquellas ideas integrales como válidas, tomando en cuenta a los subordinados, donde expresen la realidad de la institución educativa. Capacitar al personal continuamente para lograr el mejor desempeño de sus funciones, generando concursos para mejorar su conocimiento. Generar políticas encaminadas en innovar las formas de enseñanza, promoviendo el aprendizaje de las nuevas tecnologías de información y comunicación como estrategias de enseñanza lo que faciliten la verificación de los logros en los cambios de las estrategias de enseñanza. Así como comprometerse con los cambios necesarios, reconociendo cuando asumen la responsabilidad como un valor en beneficio y minimizando las distancias jerárquicas que lo separan para que asuman el compromiso de ejecutar actividades pedagógicas de calidad en la institución.

\section{REFERENCIAS}

Albrecht, K. (2006). Inteligencia Social. Barcelona, España: Editorial Vergara

Capriotti (2009). Planificación Estratégica de la Imagen Corporativa. Libro en Línea. Disponible en: www.bidireccional.net

Chiavenato, I., y González, E. A. (2017). Comportamiento Organizacional: La dinámica del éxito en las organizaciones. México DF: McGraw-Hill

Faintein, J. (2008). La Toma de Decisiones de la Empresa. Buenos Aires: Ed. Atlántida

Hellriegel, D., Jackson, S. E., y Slocum, J. W. (2002).Administración: un enfoque basado en competencias. Thomson Learning

Newstrom, J. W., Brito, M. P. G., Carrión, M. A. S., y Quiñones, A. D. (2011).Comportamiento humano en el trabajo. Ciudad de México: McGraw-Hill

Plan Decenal de Educación (2018) Ministerio de Educación Colombia. Bogotá

Polimeni, Fabozzi y Adelberg (2007) Contabilidad de costos: Concepto y aplicaciones para la toma de decisiones gerenciales. México: McGraw Hill 